\title{
OPTIMIZED PROFILES FOR IMPROVED CONFINEMENT AND STABILITY IN THE DIII-D TOKAMAK
}

by

T.S. TAYLOR, H. ST JOHN, A.D. TURNBULL, Y.R. LIN-LIU, K.H. BURRELL, V. CHAN, M.S. CHU, J.R. FERRON, L.L. LAO, R.J. LA HAYE, E.A. LAZARUS, R.L. MILLER, P.A. POLITZER, D.P. SCHISSEL, and E.J. STRAIT 


\section{DISCLAIMER}

This report was prepared as an account of work sponsored by an agency of the United States Government. Neither the United States Government nor any agency thereof, nor any of their employees, makes any warranty, express or implied, or assumes any legal liability or responsibility for the accuracy, completeness, or usefulness of any information, apparatus, product, or process disclosed, or represents that its use would not infringe privately owned rights. Reference herein to any specific commercial product, process, or service by trade name, trademark, manufacturer, or otherwise, does not necessarily constitute or imply its endorsement, recommendation, or favoring by the United States Government or any agency thereof. The views and opinions of authors expressed herein do not necessarily state or reflect those of the United States Government or any agency thereof. 


\section{DISCLAIMER}

Portions of this document may be illegible in electronic image products. Images are produced from the best available original document. 


\title{
Conf-9406270--8 \\ GA-A21757
}

\section{OPTIMIZED PROFILES FOR IMPROVED CONFINEMENT AND STABILITY IN THE DIII-D TOKAMAK}

\author{
by \\ T.S. TAYLOR, H. ST JOHN, A.D. TURNBULL, Y.R. LIN-LIU, \\ K.H. BURRELL, V. CHAN, M.S. CHU, J.R. FERRON, L.L. LAO, \\ R.J. LA HAYE, E.A. LAZARUS, ${ }^{\star}$ R.L. MILLER, P.A. POLITZER, \\ D.P. SCHISSEL, and E.J. STRAIT
}

This is a preprint of an invited paper presented at the 21st European Physical Society Conference on Plasma Physics and Controlled Fusion, June 27 through July 1,1994 , in Montpellier, France, and to be printed in the PROCEEDINGS.

Work supported by the U.S. Department of Energy

under Contract Nos. DE-AC03-89ER51114 and DE-AC05-84OR21400

*Oak Ridge National Laboratory, Oak Ridge, Tennessee.

\section{GA PROJECT 3466 \\ FEBRUARY 1995}

IJ DISTRIBUTION OF THIS DOCUMENT IS UNLIMITED GENERAL ATOMIES 


\title{
Optimized profiles for improved confinement and stability in the DIII-D tokamak
}

\author{
T.S. Taylor, H. St. John, A.D. Turnbull, Y.R. Lin-Liu, K.H. Burrell, V. Chan, M.S. \\ Chu, J.R. Ferron, L.L. Lao, R.J. La Haye, E.A. Lazarus, R.L. Miller, P.A. Politzer, \\ D.P. Schissel, EJ. Strait
}

General Atomics, P.O. Box 85608, San Diego, California 92186-9784

\begin{abstract}
Simultaneous achievement of high energy confinement, $\tau_{\mathrm{E}}$, and high plasma beta, $\beta$, leads to an economically attractive compact tokamak fusion reactor. High confinement enhancement, $H=\tau_{E} / \tau_{E-I T E R} 89 P=4$, and high normalized beta $\beta_{N}=$ $\beta /(\mathrm{I} / \mathrm{aB})=6 \%-\mathrm{m}-\mathrm{T} / \mathrm{MA}$. have been obtained in DIII-D experimental discharges.

These improved confinement and/or improved stability limits are observed in several DIII-D high performance operational regimes: VH-mode, high $\ell_{i} \mathrm{H}$-mode, second stable core, and high beta poloidal. We have identified several important features of the improved performance in these discharges: details of the plasma shape, toroidal rotation or $E x B$ flow profile, $q$ profile and current density profile, and pressure profile. From our improved physics understanding of these enhanced performance regimes, we have developed operational scenarios which maintain the essential features of the improved confinement and which increase the stability limits using localized current profile control. The stability limit is increased by modifying the interior safety factor profile to be nonmonotonic with high central $q$, while maintaining the edge current density consistent with the improved transport regimes and the high edge bootstrap current. We have calculated high beta equilibria with $\beta_{\mathrm{N}}=6.5$, stable to ideal $n=1$ kinks and stable to ideal ballooning modes. The safety factor at the $95 \%$ flux surface is 6 , the central $q$ value is 3.9 and the minimum in $q$ is 2.6 . The current density profile is maintained by the natural profile of the bootstrap current, and a modest amount of electron cyclotron current drive.
\end{abstract}

\section{Introduction}

In many tokamak reactor designs, the conditions necessary for ignition are gained primarily by increasing the plasma current: energy confinement varies approximately linearly with plasma current, $\tau_{E} \propto I$, and the $\beta$ limit is proportional to the normalized current, $(\mathrm{I} / \mathrm{aB}), \beta_{\mathrm{T}}^{\max } \propto \mathrm{I} / \mathrm{aB}$. However, common confinement scalings, such as the ITER-89P confinement scaling (Yushmanov 1990) lead to very large and costly tokamak reactor designs. Significant reductions in the size of the reactor core, the capital cost of the reactor core, and the net cost of electricity can be realized if both the confinement and the $\beta$-limit can be increased (Galambos 1994). Further improvements in the tokamak reactor concept can be made if these improvements in $\beta_{\mathrm{T}}^{\max }$ and $\tau_{\mathrm{E}}$ are obtained in steady-state discharge conditions with a high bootstrap current fraction (Kikuchi 1993).

Many tokamak experiments are now routinely observing confinement times which far exceed that predicted by the ITER-89P scaling, often by factors of 3 to 4 . For example, in DIII-D high values of $H(H=$

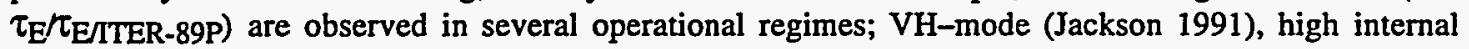
inductance $\left(\ell_{i}\right) \mathrm{H}$-mode (Lao 1993a), and high poloidal $\beta$ (Politzer 1994). The performance in the high confinenent regimes observed in the JET, JT-60U, TFTR, and DII-D tokamaks is no longer limited by transport or heating power, but instead are limited by stability limits at high $\beta_{T}$ (JET Team 1993, Mauel 1993, Zarnstorff 1993, Taylor 1993, Strait 1993). Performance in present day tokamalis can be improved if the $\beta$-limit can be increased while maintaining the observed high confinement. Our strategy for identifying a self-consistent high confinement high beta steady-state discharge scenario is to identify and maintain those features that are favorable for the high confinement and then modify the profiles to increase the stability limit, without adversely affecting the confinement. The features that we have identified that are favorable for high confinement are: (1) strong plasma shaping, high triangularity $(\delta)$ and high elongation $(\kappa) ;(2)$ high plasma rotation, large shear in the $E \times B$ flow; (3) finite current density near the edge; (4) negative central shear; and (5) high $q(0)$. We intend to show are compatible with steady-state high $\beta$.

In the next section, we review a number of high performance regimes that have been identified in DIII-D experimental discharges, and discuss the features that we believe are important for achieving the high 
performance. The profile considerations for achieving high confinement and the consistency with high beta are discussed. In Section 3 we identify a possible solution that allows for both high confinement and stability at high beta. We show transport simulations that indicate this high beta, high confinement regime can be obtained and sustained in future DIII-D with modest amounts of localized current drive in Section 4. We discuss these results and draw some conclusions in Section 5.

\section{Experimentally Achieved Improved Confinement Regimes}

Four regimes of improved plasma performance have been identified in DIII-D: these are high $\ell_{\mathrm{i}} \mathrm{H}$-mode discharges (Lao 1993a, b; Ferron 1993), second stable core discharges (Lazarus 1992), VH-mode discharges (Jackson 1991, Osborne 1994, Taylor 1992, Greenfield 1993), and high beta poloidal discharges (Politzer 1994). We have identified several discharge features that are conducive to high confinement. These features are details of the plasma shape (Lazarus 1993, Hyatt 1994), the toroidal rotation and sheared ExB flow (Groebner 1993, Burrell 1994), and details of the current density or $q$ profile. The current density profile or $q$ profile features that are important include high $\ell_{\mathfrak{i}}$, finite current near the edge (Ferron 1994), and negative central shear.

\subsection{Discharge shape}

The highest confinement times in DIII-D discharges are obtained in strongly shaped plasmas with high triangularity, $\delta$, and high elongation, $K$. The dependence of energy confinement time on the triangularity was first clearly noted in VH-mode discharges (Taylor 1993, Osborne 1994). The energy confinement time increased with triangulari)ty in these discharges, and at the lowest triangularity, $\delta=0.35$ in single-null discharges (SND, VH-mode conditions were not obtained. Recently, we completed a detailed evaluation of the plasma performance on the shape of the plasma (Lazarus 1993, Lazarus 1994, Hyatt 1994); four discharge shapes were optimized and studied in detail. As shown in Fig. 1, the discharge performance (as measured by the product $\beta \tau$ ) of the low triangularity discharges is not very high and increasing the elongation at low triangularity is not very effective at improving the performance. There is a substantial increase in the performance when the plasma triangularity is increased, and at high triangularity, increasing the elongation does result in a further increase in plasma performance. In both the high triangularity shapes, VH-mode was obtained; all features characteristic of VH-mode were observed including a clearly observable "spin-up" and an increase in the $E x B$ shear flow in the region of $0.6<\rho<0.9$. (Osborne 1994).

The high confinement regimes are also accessible at higher current as a consequence of strong shaping. In all large tokamaks, the highest performance, as measured by $\beta \tau$ or $n \mathrm{~T}_{\mathrm{i}} \tau$, is observed at $q_{95} \geq 4$. In DII-D, the highest performance is observed at $995 \sim 4.5$ and the highest normalized performance, which we define as $\beta_{\mathrm{NH}}$, is at $q 95 \approx 6$. However, we observe an increase in absolute performance with plasma current, as expected from prevalent confinement scaling laws, for $q>4$. Higher triangularity and higher elongation allow higher plasma current with a given safety factor $q$. The increase in the performance from smaller elongation to higher elongation in Fig. 1 is primarily a consequence of the increase in the capability of the shape to carry more current.

An important consequence of the strong shaping is the improvement in confinement at high $\beta$. In low triangularity discharges, especially low triangularity single-null divertors, the confinement begins to degrade as $\beta \mathrm{N}=\beta /(\mathrm{I} / \mathrm{aB})$ exceeds $2 \%$-m-T/MA. In the higher triangularity discharges, the high confinement regime extends to higher $\beta$, up to $\beta_{\mathrm{N}}$ nearly $4 \%$-m-T/MA (Lazarus 1993).

\subsection{Plasma rotation and sheared ExB flow}

Sheared ExB flow has been identified as important cause of the reduced turbulence and reduced transport in tokamaks (Biglari 1990, Shaing 1990, Groebner 1993, Burrell 1992, Burrell 1994). The increase in the sheared ExB flow in the edge region is the leading paradigm for the reduced transport and increased confinement in $\mathrm{H}$-modes compared to $\mathrm{L}$-modes; there exists a large amount of experimental and theoretical support for this model. Stabilization of microturbulence by sheared $E x B$ flow is also the leading candidate to explain the improved confinement in VH-mode discharges. There is a decrease in the transport and the measured turbulence in VH-mode discharges at the same spatial location $(0.6<\rho<0.9)$ and at the same time in the discharge as the observed increase in the shear in the $E x B$ flow (Osborne 1994).

We have recently completed experiments on DIII-D using magnetic braking as an independent control of the plasma rotation, to verify that the plasma rotation and the $E x B$ flow play a major role in the reduction of the transport (La Haye 1994, Burrell 1994). It has been shown experimentally and theoretically (Hender 1989, Jensen 1983) that an imposed helical field interacts nonlinearly with the plasma and will slow the plasma rotation - too large an external field results in "locked modes." In DIII-D discharges, an external 


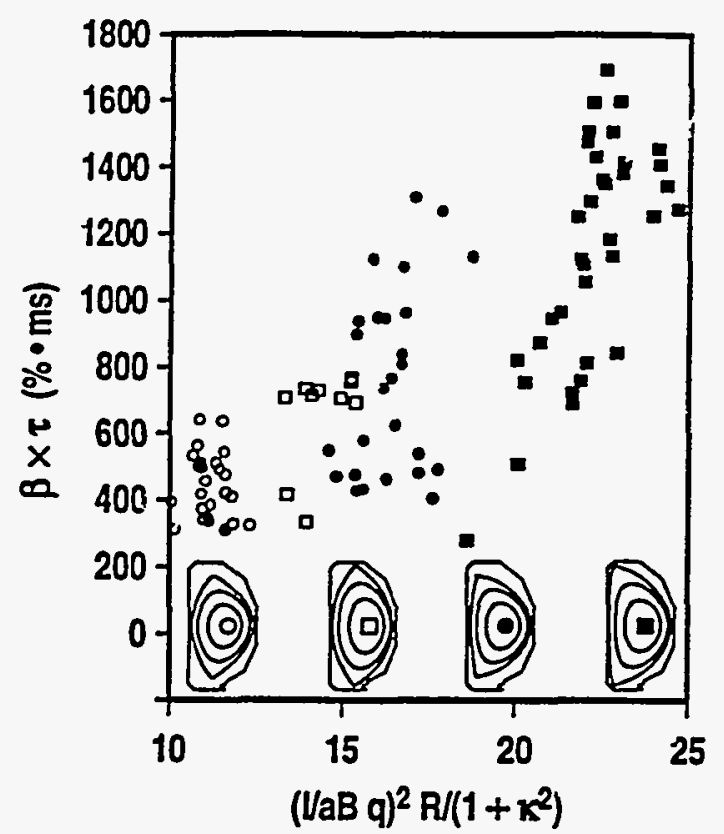

Fig. 1. Dependence of plasma performance on discharge shape: $O=\kappa=1.7, \delta=0.3 ; 0=$ $\kappa=2.1, \delta=0.3 ; O=\kappa=1.7, \delta=0.9 ; \square=$ $\kappa=2.1, \delta=0.9$.

coil was used to impose a helical field and substantially reduce the plasma toroidal rotation in both high $\ell_{\mathrm{i}}$ H-modes (Lao 1993a,b) and VH-modes (La Haye 1994). This procedure allows direct comparison of discharges that are very similar in all respects except for the plasma rotation profiles. An example of such a comparison for VH-mode discharges is shown in Fig. 2. In both cases, the discharges evolved similarly except for the application of the external helical field. For the case depicted by the dashed line, the external coil was energized during the high confinement phase, resulting in lower central rotation and radial electric field, Fig. 2(a), substantial reduction in the shear in the $E x B$ flow, Fig. 2(b), and a substantial increase in the transport, Fig. 2(c). The results in the high $\ell_{i} H$-mode were similar to those shown here, indicating that the reduction of transport in both $\mathrm{VH}$-mode discharges and high $\ell_{\mathrm{i}} \mathrm{H}$-mode discharges is largely a consequence of the increased sheared $E x B$ flow.

Although much less well documented, it is becoming increasingly apparent that the plasma rotation and the plasma rotational shear are very important for MHD stability. Plasma rotation relative to a resistive wall is well known to have the same effect as a perfectly conducting wall for resistive plasma tearing modes (Jensen 1983). Recent theoretical work has shown that plasma rotation with respect to a resistive wall can improve stability against low $\mathbf{n}$ kink modes at high beta (Bondeson 1994). Also, shear in the rotation can improve stability against ideal ballooning modes (Miller 1994) and resistive MHD modes. Recent experiments on DII-D have documented the achievement of high beta in regimes where the beta limit is expected to be much lower in the absence of wall stabilization, and these experimental results clearly show that plasma rotation is important (Strait 1994, Turnbull 1994a). High beta was obtained at low $q$, low $\ell_{i}$ discharge. $\left(\beta_{\mathrm{T}}=12.5 \%, q_{95}=2.5, \ell_{\mathrm{i}}=0.72, \beta_{\mathrm{N}}=4.4 \%-\mathrm{m}-\mathrm{T} / \mathrm{MA}\right)$. Previous ideal MHD theory and calculations indicate that without strong wall stabilization, $\beta_{\mathrm{N}}$ should increase with $\ell_{\mathrm{i}}, \beta_{\mathrm{N}}=4.4 \ell_{\mathrm{i}}$ (Lao $1993 \mathrm{~b})$. Therefore, much lower values of $\beta_{\mathrm{T}}$ and $\beta_{\mathrm{N}}$ were expected than were achieved, suggesting the role of wall stabilization.

The importance of plasma rotation in achieving high beta is shown in the details of the termination of a high $\beta$ discharge. $q(0)$ was maintained above 1 by heating the discharge with neutral beam injection before the current end of rampup to allow easier interpretation of the stability anlaysis. In this discharge, $\beta_{\mathrm{N}}=3.7 \%$ $\mathrm{m}-\mathrm{T} / \mathrm{MA}$ was reached (Fig. 3). Ideal stability analysis with the code GATO has been completed for this discharge using exact experimental equilibria reconstructed with the measured pressure profile and the measured $q$ profile from motional Stark effect measurements (Wroblewski 1993). The highest stable beta 
against low $\mathrm{n}$ kink modes with no wall stabilization is $\beta_{N}=2.8 \%-m-T / M A$; the experimental equilibria are calculated to be stable against low $n$ kinks if wall stabilization is included. This analysis indicates that a $30 \%$ gain in the stability limit is provided by the wall. Higher beta is prevented by the appearance of TAE modes which result in a loss of heating efficiency. A rotating $\mathrm{m} / \mathrm{n}=5 / 2$ mode is observed during the discharge; starting before the high $\beta$ phase. Approximately $10 \mathrm{~ms}$ prior to the plasma termination, a nonrotating $m / n=3 / 1$ mode begins to grow very rapidly, while the $m / n=5 / 2$ mode continues to rotate. The growth rate of the $3 / 1$ mode is $\gamma=(6 \mathrm{~ms})^{-1}$ consistent with the expectation for a resistive wall mode (Bondeson 1994). At the time the $\mathrm{m} / \mathrm{n}=3 / 1$ mode begins to grow rapidly, the measured rotation from charge exchange recombination measurements (Gohil 1992)] becomes zero. It is expected that if the rotation could be maintained significantly different than zero at the $3 / 1$ surface, higher $\beta$ could be obtained.

\subsection{Details of the current density and $q$ profile}

Several features of the current density and $q$ profile have been shown to be important for improving performance. In a number of tokamaks, both confinement and achievable $\beta$ have been shown to increase with increasing internal inductance (Lao 1993a, b; Ferron 1993; Strait 1994a). In DIII-D we have shown that the current density near the edge plays a role in achieving high confinement in certain operating regimes (Ferron 1994) and is in general unfavorable for achieving high beta (Strait 1993, Taylor 1991). A region near the axis with negative central shear allowing access to the second stability regime was identified in DIII-D experiments as favorable for high beta and very high central beta, and improved transport with a central negative shear has been shown in several experiments (Hugon 1992, Tubbing 1991, Hoang 1994). Obtaining elevated central $q$ values substantially greater than unity have been shown to reduce central transport in DII-D high beta poloidal discharges (Politzer 1994) and may be important for achieving the best performance in DIII-D VH-mode discharges.

Both $\tau_{\mathrm{E}}$ and $\beta_{\mathrm{T}}^{\max }$ have been shown to increase with increasing internal inductance, $\ell_{\mathrm{i}}$, ${ }^{\text {although a }}$ self-consistent steady-state high $\ell_{i}$ scenario has not yet been identified at high $\beta_{\mathrm{T}}$. Several experiments have reported the use of negative current ramps and elongation ramps to transiently increase $\ell_{\mathrm{i}}$ and $\tau_{\mathrm{E}}$ (Zarnstorff 1992). In DII-D ELMing $H$-mode discharges $H=3.8$ was achieved. Both theory and experimental results show that the $\beta$-limit increases with increasing $\ell_{i}$ and the maximum achievable $\beta$ consistent with DII-D experiment is given by $\beta_{\mathrm{T}}^{\max } \leq 4 \ell_{\mathrm{i}} \mathrm{I} / \mathrm{aB}$ (Tumbull 1986, Howl 1992, Taylor 1991, Lao 1993b). In DIII-D near circular discharges, $\ell_{i}$ was varied using different negative current ramp rates, and the $\beta$-limit was shown to scale linearly with $\ell_{\mathrm{i}}$ in good agreement with the calculated ballooning limit. At $\ell_{\mathrm{i}} \sim 2, \beta_{\mathrm{N}}=6 \%-\mathrm{m}-\mathrm{T} / \mathrm{MA}$ was obtained (Ferron 1993).

The increase in both $\tau_{\mathrm{E}}$ and $\beta_{\mathrm{T}}^{\max }$ with increasing $\ell_{\mathrm{i}}$ would seem to make the high $\ell_{\mathrm{i}}$ discharge scenario a very attractive high performance scenario. However, high $\beta_{\mathrm{T}}$ was obtained at high $\ell_{\mathrm{i}}$ using transient techniques and as the current profile relaxes towards a steady-state profile both $\ell_{\mathrm{i}}$ and $\beta_{\mathrm{T}}^{\max }$ decrease. The decrease in $\ell_{\mathrm{j}}$ is a consequence of the radial distribution of the bootstrap current. The bootstrap current density is proportional to $\sqrt{\varepsilon} p^{\prime}$, where $\varepsilon=r / R_{0}$ is the inverse aspect ratio, and $p^{\prime}$ is the pressure gradient (Kikuchi 1990). At high $\beta, p^{\prime}$ near the boundary is always large and in most cases approaches the value marginally stable to ballooning. So at high $\beta$, fully relaxed profiles will include high bootstrap current near the plasma edge. Preliminary transport and current drive modeling of high $\beta$ scenarios confirm the difficulty

${ }^{*}$ Here $\ell_{\mathrm{i}}=(1 / \mathrm{V}) \int \mathrm{d} v \mathrm{~B}_{\mathrm{P}}^{2} /\left(\mathrm{B}_{\mathrm{PA}}^{2}\right)$, where $\mathrm{BPA}_{\mathrm{PA}}=\mu_{0} \mathrm{I} / \mathrm{CP}_{\mathrm{P}}, \mathrm{CP}_{\mathrm{P}}$ is the poloidal circumference. 
in realizing high $\ell_{\mathrm{i}}$. With centrally peaked current drive only, the maximum $\ell_{\mathrm{i}}$ calculated with $\beta_{\mathrm{N}}=5$ and $q 95=8$ is $\ell_{\mathrm{i}}=1.2-1.4$ depending on the details of the model. In experiments in strongly shaped discharges high $\beta$ has been obtained with only moderate values of $\ell_{\mathrm{i}}: \beta_{\mathrm{N}}=5$ at $\ell_{\mathrm{i}}=1.2$ and $\mathrm{q} 95=5$ has been obtained in DIII-D. It remains a challenge to verify high confinement at these values of $\ell_{\mathrm{i}}$ and $\beta_{\mathrm{N}}$. We will not therefore include high $\ell_{\mathrm{i}}$ in a feature for a high performance steady-state scenario.

Recent experiments in DIII-D ELMing H-mode and VH-mode discharges have shown that current density near the edge has a favorable impact on confinement in these discharges although this current density leads to lower $\ell_{\mathrm{i}}$. In $\mathrm{H}$-modes and $\mathrm{VH}$-modes significant bootstrap current is calculated near the edge as a consequence of the high edge pressure gradient. The high confinement observed in VH-modes in JET and DII-D is correlated to the local access to the second regime of stability to ballooning modes near the edge, afforded by the high edge current density (Greenfield 1993). Negative current ramps are calculated to reduce the current density near edge on a short time scale. In Fig. 4, we show the effect of negative current ramps on confinement. There are four discharges shown with different negative current ramp rates. The chain dash curve indicates a standard VH-mode discharge with the plasma current held constant: the maximum value of $H$ is near 3.5, a typical value of DIII-D VH-mode discharges. With a slight negative current ramp, $d V / d t=$ $-0.5 \mathrm{MA} / \mathrm{s}$, the confinement improves to $H \cong 3.9$. $\beta_{\mathrm{N}}$ reached $3.5 \%-\mathrm{m}-\mathrm{T} / \mathrm{MA}$ for this case. Increasing the magnitude of negative current ramp, $d l / d t=-0.8 \mathrm{MA} / \mathrm{s}$, also shows good confinement and the increase to high values of $H$ has less of a time delay following beam injection. However, the high confinement for the $-0.8 \mathrm{MA} / \mathrm{s}$ case is very short even though $\ell_{\mathrm{i}}$ is higher and $\beta_{\mathrm{N}}$ is lower, $\beta_{\mathrm{N}}=2$, than the previously mentioned cases. Finally, at $d L / d t=-1 \mathrm{MA} / \mathrm{s} \mathrm{VH}-$ mode is not obtained and $H$ remains approximately 2 even though $\ell_{\mathfrak{i}}$ reaches a high value, $\ell_{i}=2.3$. Furthermore, the highest confinement enhancement, $H$, in this discharge occurs not at the highest $\ell_{\mathrm{i}}$ but a little later, after the negative surface voltage is removed and $\ell_{\mathrm{i}}$ begins to decrease. This relation between negative current ramps and the confinement is only observed in discharges with a substantial edge pressure gradients; and in ELMing $\mathrm{H}$-modes, it is clear that with a sufficiently high negative voltage, the edge pressure pedestal is lost (Ferron 1994).

One aspect of the $q$ profile and current density profile that has been shown to be very beneficial to stability is a region of negative central shear, and the consequent access to second stability. Negative central shear with a second stable core were first observed in DIII-D high beta discharges (Lazarus 1991, Lazarus 1992). The negative central shear region was produced by strong heating and ramping the discharge elongation up to $K=2.5$; the resulting $q$ profile and pressure profile are shown in Fig. 5. The central beta reached $44 \%$, and the volume average beta was $\beta_{\mathrm{T}}=$ $11 \%$. In addition to the second regime access and the high central beta, this experiment taught us two other important aspects concerning stability; 1) two low order rational, $n=1$, surfaces can co-exist without catastrophic reconnection, and 2) the existence of these two $n=1$ surfaces does not necessarily negatively impact the maximum achievable beta; $\beta_{\mathrm{T}}=$ $11 \%$ was maintained for $50 \mathrm{~ms}$. It has been speculated that these two aspects might be a consequence of the substantial separation of the two rational surfaces and the relatively strong magnetic shear at both rational surfaces. These characteristics will figure strongly in defining a stable high performance scenario. The negative central shear also has a positive impact on transport. Experimenters on JET (Tubbing 1991, Hugon 1992) and Tore Supra (Hoang 1994) have observed reduced core transport in discharges with negative central shear.

There is experimental evidence in DIII-D discharges, that $q(0)$ substantially greater than unity is favorable to reducing core transport and improving stability to ideal ballooning modes. Several early experiments and theory calculations show the benefit of higher $q(0)$ in obtaining access to the second stability regime over the entire discharge (Simonen 1988, Gerver 1988, Chance 1991). Recent experiments in

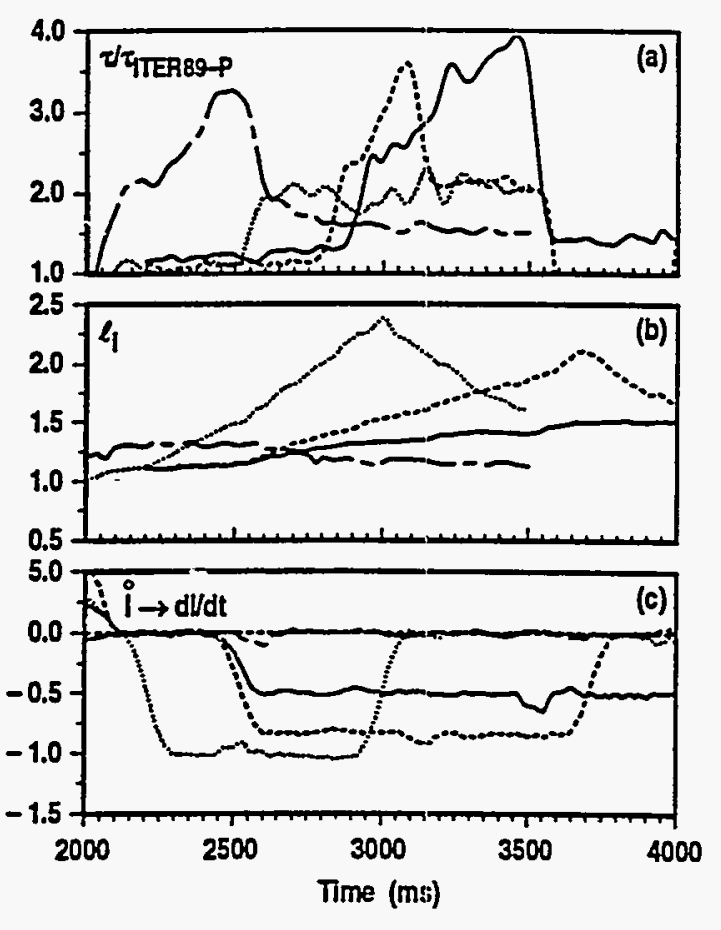

Fig. 4. Dependence of confinement enhancement on magnitude of negative current ramp: chain dash is standard VH-mode, $\dot{\mathrm{I}}_{\mathrm{p}}=0$; solid is $\dot{\mathrm{I}}_{\mathrm{p}}=$ $-0.5 \mathrm{MA} / \mathrm{s}$, dashed is $\dot{\mathrm{I}}_{\mathrm{p}}=-0.8 \mathrm{MA} / \mathrm{s}$, dotted is

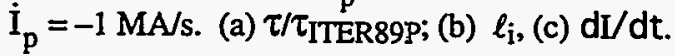




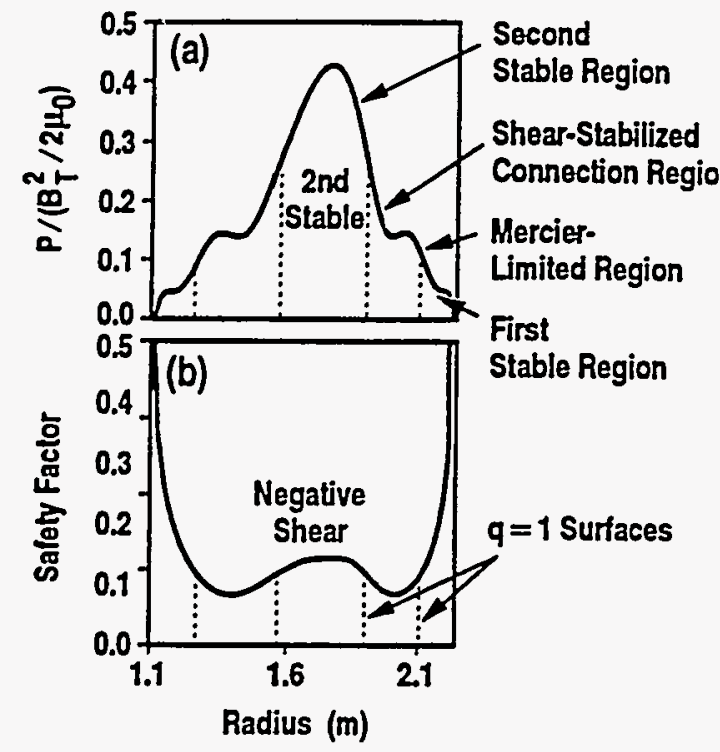

Fig. 5. DIII-D second stable core high beta: (a) reconstructed pressure profile; (b) reconstructed $q$ profile.

DIII-D have verified the global access to the second stability regime and have also demonstrated a regime of reduced core transport with $q(0)>2$ (Politzer 1994, Casper 1994). The temporal evolution of a high beta poloidal discharge is shown in Fig. 6. Following beam injection at $1 \mathrm{~s}$, the central $q$ value, measured by motional stark effect (Wroblewski 1993) continues to increase [Fig. 6(a)]. Notice also $\ell_{\mathrm{i}}$, near 2 before beam injection, decreases to about 1.3 [Fig. 6(b)] illustrating the difficulty in maintaining a high $\ell_{\mathrm{i}}$ in near steady-state conditions. At approximately $3 \mathrm{~s}, q(0)$ increases above 2 , the MHD activity disappears [Fig. $6(\mathrm{c})$ ] and the transport decreases as evidenced by the increase in central $n_{\mathrm{e}}$ Fig. 6(d)] and in central $T_{e}$ [Fig. 6(e)].

\section{The second stable core VH-mode}

We have identified a high performance scenario with profiles that are consistent with high confinement, high $\beta$, and steady-state, obtainable with a modest amount of localized current drive. The basic ideas have been reported elsewhere (Chan 1993, Taylor 1993, Turnbull 1994b) but here we describe a more complete selfconsistent scenario with slight modification of some of the details. We start with the conditions of VH-mode, observed in DII-D. The VH-mode has confinement more than sufficient for high performance, $H>3$, and the current density profile and pressure profile are close to what we expect in high $\beta$ steady-state conditions. In this scenario, we maintain the strong plasma shape, $\delta=0.8, \kappa=2.1$. The high plasma rotation and rotational shear observed in DIII-D VH-modes are expected to be favorable for high $\beta$ stability with the resistive wall as well as provide stabilization of turbulence for the improved confinement. We allow for large current density near the edge, the bootstrap current: we see no way to avoid this large edge bootstrap current at high $\beta$, and have determined no effective steady-state current drive scheme to remove it. The high $\beta$ stability limit is increased by modifying the interior safety factor profile to be non-monotonic with high central $q, q(0)=3.9$ and $q_{\min }=2.6$. The negative central shear is similar to that observed in DII-D second stable core high $\beta$ discharges, and so we have called this high performance scenario "second stable core VH-mode" (SSC VH-mode) (Turnbull 1994b).

The increase in the $q(0)$ and the negative central shear has a number of motivations. Most importantly, the resultant current density and $q$ profile provide stability to low $n$ modes to high values of $\beta_{\mathrm{T}}$. High confinement discharges at high beta have finite p' at the boundary, which results in substantial edge bootstrap current. With $q(0)$ near 1, the high edge pressure gradient is destabilizing to low $\mathbf{n}$ kink modes (Strait 1993, 
Ferron 1994). By raising $q(0)$ and $q_{\min }$ above low order rational surfaces, the stability limit is increased. Previous $\beta$ optimization studies (Turnbull 1989) in conjunctions with the straight tokamak model (Friedberg 1987, Wesson 1978), suggest this promising approach to improving stability. To lowest order, the stabilizing energy is proportional to $(m-n q)^{2}$. To improve stability, surfaces where this term vanishes should be eliminated as much as possible, and if the low order rational surfaces can not be eliminated, the volume where the stabilizing term $(m-n q)^{2}$ vanishes should be kept as small as possible. In other words the low order rational surfaces which can not be eliminated should be in a region of high magnetic shear. The most dangerous MHD modes, $\mathrm{m} / \mathrm{n}=1 / 1,3 / 2,2 / 1$, and $5 / 2$ can be entirely eliminated by raising $q$ everywhere above 2.5. Stabilization of other modes is provided by ensuring that the shear near the remaining rational surfaces is large, either positive or negative. It is important to note that the second stable core high beta discharges exhibited this negative central shear region, $q(0) \approx 1.1$ and $q_{\min } \approx 0.85$, and remained stable for several energy confinement times, with two separate and saturated $n=1$ modes (Lazarus 1991, Lazarus 1992). Improved stability with $q_{\min }>2.5$ is expected to be much easier. Theories have also been developed which predict improved confinement (Kessel 1994).

In any steady-state high performance scenario, the current drive requirements are a concern. The second stable core VH-mode current profile has bootstrap current density profile similar to that of the final target profile. To obtain the inverted shape of the $q$-profile, modest localized current drive near the half radius is required. Electron cyclotron current drive, ECCD, is ideal for this task of localized current drive.

\section{Modeling the second stable core VH-mode}

In this section we describe the details of the self consistent modeling of the second stable core VH-mode. To evaluate the stability boundary for kink instabilities and to evaluate the impact of the proximity of the wall on the stability, we generated a set equilibria using the pressure profile from the transport analysis for the case $\beta_{N}=5.7 \%-m-T / M A$. The current profile and the $q$ profile are shown in Fig. 7(b); note the high current density near the edge. The plasma shape was maintained fixed; $R_{0}=1.68 \mathrm{~m}, \mathrm{a}=0.62 \mathrm{~m}$, $\kappa=2.1, \delta=0.8$; the toroidal field was constant at $\beta_{\mathrm{T}}=1.95 \mathrm{~T}$; the plasma current was constant at $\mathrm{I}=$ 1.6 MA, and $q_{0}$ and $q_{\min }$ were held fixed at 3.9 and 2.6 respectively. The details of the $q$ profile and the current profile varied roughly consistent with transport as $\beta$ increased: in particular as $\beta_{\mathrm{N}}$ increased from 2.4 to $6.5 \%-\mathrm{m}-\mathrm{T} / \mathrm{MA}, \ell_{\mathrm{i}}$ decreased from 0.74 to 0.55 and the edge current density increased from $J_{95} /\langle\mathrm{J}\rangle=0.23$ to 0.64 , where $J_{95}$ is the flux surfaced average current density at the 95\% flux and $\langle J\rangle$ is the total plasma current divided by the total poloidal cross sectional area. Both the increase in edge current and decrease in $\ell_{i}$ are in general destabilizing to low $n$ kinks at high $\beta$.

The stability limit to low $n$ kink modes depends critically on the stabilizing influence of the DIII-D vessel wall, as shown in Fig. 7. Using the equilibria as described above, we varied the DIII-D wall position by uniformly increasing the distance from the plasma to the wall keeping the wall shape fixed. The location of the DIII-D wall with respect to the last closed flux surface is shown as an inset in Fig. 7, as well as the case where the wall radius was increased by a factor of 2 . The shape of the wall is not conformal to the plasma, but for the plasma shape shown the wall to plasma distance is $r_{w a l l} / a=1.3$. Low $n$ mode ideal MHD stability was calculated with the code GATO (Bernard 1981). As can be seen clearly in

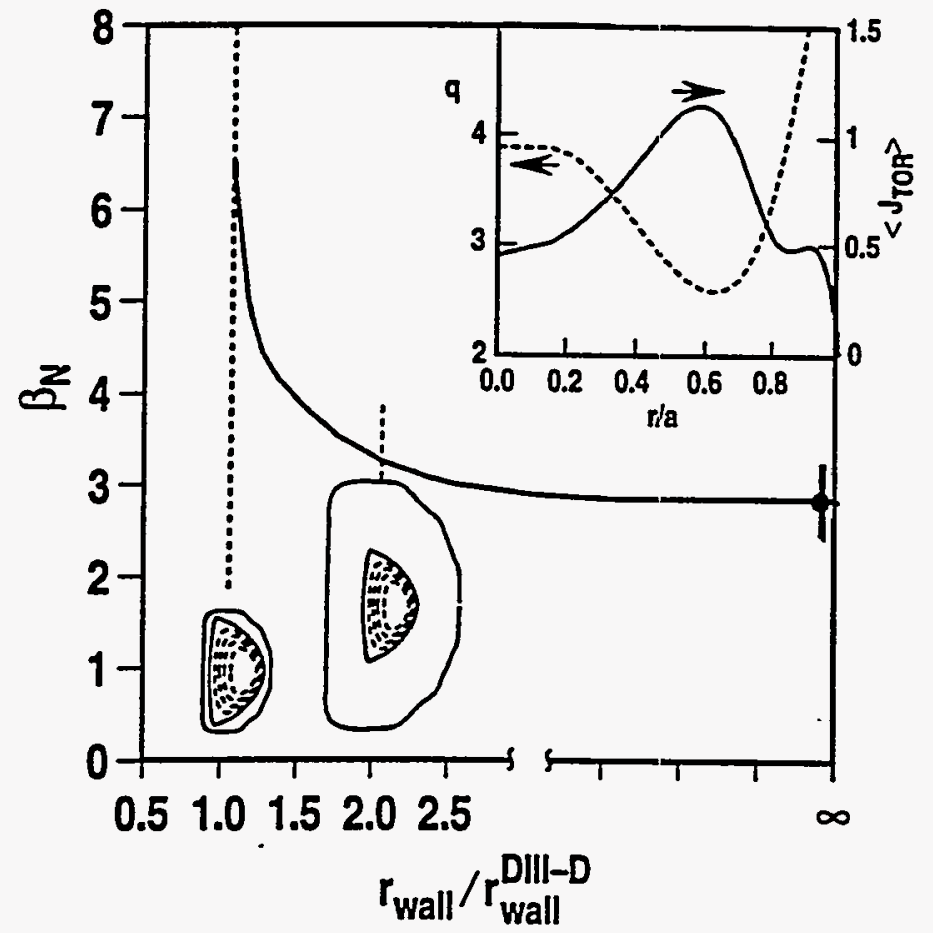

Fig. 7. Dependence of the calculated $\beta$-limit on plasma to wall distance. (b) (inset) $q$-profile and current profile for $\beta_{\mathrm{N}}=$ 5.7\%-m-T/MA. 
the figure, the maximum beta stable to the $n=1$ mode decreases rather rapidly as the wall is moved further from the plasma, and with no wall stabilization is only $\beta_{\mathrm{N}}=2.8$. Therefore, for such a configuration to achieve high beta, stabilization with a resistive wall must be demonstrated and fully understood, and provision for maintaining rotation and rotational shear must be included (Turnbull 1994a, Bondeson 1994, Strait 1994b) - a strong motivation for maintaining neutral beam injection capability. With a conducting wall at the location of the DIII-D vessel, this scenario is stable against both $n=1$ and $n=2$ modes up to $\beta_{\mathrm{N}}=5.7 \%-\mathrm{m}-\mathrm{T} / \mathrm{MA}$. Nearly the entire plasma is in the second stable regime to ideal ballooning modes, however a small region near the boundary is in the first regime and is marginally stable to ballooning modes. Ballooning mode stabiity is evaluated with the CAMINO code (Chance 1987).

To carry out the transport calculations, a suitable but simple transport model is required. The data base for high power heating with fast wave heating, and electron cyclotron heating is limited, so we have chosen for the electron transport, the INTOR model (Wesson 1987), with a profile modification, $\mathrm{K}_{e}=\mathrm{n} \chi_{e}=$ $5 \times 10^{19} /\left(1-0.8 \rho^{2}\right) \mathrm{m}^{-1} \mathrm{~s}^{-1}$. The increase in the conductivity at the edge is necessary due to the otherwise extraordinarily high electron pressure gradients calculated with the very broad density profiles assumed. For the ion conductivity, we used a factor times neoclassical $\left(\mathrm{K}_{\mathrm{i}}=\mathrm{n}_{\mathrm{i}} \chi_{\mathrm{i}}\right) \chi_{\mathrm{i}}=\alpha \chi_{\mathrm{i}}^{\text {neo }}$ where the factor for the case we will show is $\alpha=2.6$. The magnitude of the transport (the constant in the electron conductivity and the multiplier for the ion conductivity) is adjusted to give an energy confinement of 3.3 times ITER89P. This choice of conductivity gives temperature profiles that are representative of VH-mode and high confinement (high $\ell_{\mathrm{i}}$ ) $\mathrm{H}$-modes. The density profile is chosen to be very similar to that of a DII-D VH-mode discharge and is held fixed in the calculations.

The transport calculations are carried out with the code ONETWO (St. John 1993) coupled to two if packages FASTWAVE (Chiu 1989), for calculating the fast wave current drive, and TORAY (Matsuda 1989), for calculating the electron cyclotron current drive. FW is used for heating and axial current drive. Neutral beam injection is used for ion heating and core current drive. ECCD is used for off axis current drive. The geometry of the DIII-D system is used for the three heating and current drive systems. The location of the ECCD current drive is changed by locating the resonance vertically above the midplane, using a moveable mirror, and the magnitude of the ECCD is controlled by adjusting the power.

The profile of the electron and ion conductivities are shown in Fig. 8(a) and the resultant temperature profiles and density profile are shown in Fig. 8(b). One important feature of these profiles is the high ion conductivity in the center. This is a consequence of the high $q(0)$ and reduced poloidal field near the axis compared to the conventional profiles with $q(0)$ near unity. In the outer portion of the discharge, the electron conductivity is higher and dominantly figures in the determination of the total confinement. From our experience, one of the essential features in defining the conductivities is the effect on the bootstrap current near the boundary and since near the boundary the bootstrap current depends on the total pressure gradient, it is the effective conductivity that is important. We have chosen the higher electron conductivity to be conservative, since the critical current drive tool is ECCD the efficiency of which improves with electron temperature. Note that the density profile is very broad with a line average of $5.7 \times 10^{19} \mathrm{~m}^{-3}$ and a value on the last closed flux surface of $2 \times 10^{19} \mathrm{~m}^{-3}$

In the code ONETWO the current density profile is calculated self-consistently with a fixed boundary equilibrium. The pressure gradient and the current density profile are calculated at each step, and the equilibria are recalculated as needed. The resultant
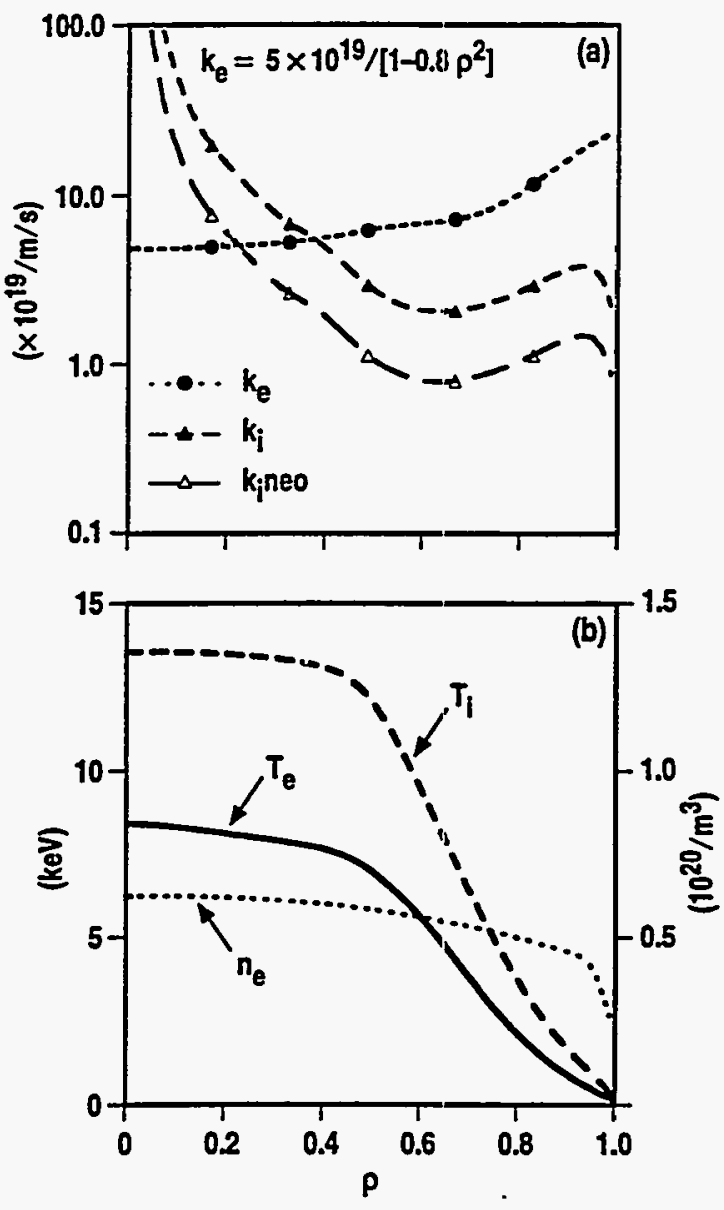

Fig. 8. Second stable VH-mode kinetic profiles. (a) Assumed electron conductivity, $\Delta$ assumerd ion thermal conductrivity, $\Delta$ neoclassical ion thermal conductivity; (b) A electron density profile, dashed line is calculated ion temperature profile, solid line line is calculated electron temperature profile. 
current profile is shown in Fig. 9. The total current is 1.6 MA. The bootstrap current accounts for $1.04 \mathrm{MA}$ or $65 \%$ of the total current and is very broad. The NBCD accounts for $0.17 \mathrm{MA}$ and is peaked near the center. A small amount of axial FWCD, or ECCD can control $q(0)$. But, because of the difficulty in avoiding stagnation points in the $q$ profile near the axis, we have let $q(0)$ rise in this case. $q(0) \sim 7$ on axis and the resultant profile is stable to $n=1$ and $n=2$ kinks with $\beta_{\mathrm{T}}=7.1 \%$ and $\beta_{\mathrm{N}}=5.5 \%-\mathrm{m}$ T/MA. Stability calculations have shown that the value of $q(0)$ as long as there is significant shear at the low order rational surfaces. The critical feature of the current profile is provided by ECCD with $0.32 \mathrm{MA}$ driven off axis to achieve the inverted $q$ profile. The total power for this scenario is $20 \mathrm{MW}, \mathrm{P}_{\mathrm{ECH}}=7 \mathrm{MW}, \mathrm{P}_{\mathrm{FW}}=6.5 \mathrm{MW}$, $\mathrm{P}_{\mathrm{NBI}}=6.5 \mathrm{MW}$, and the confinement enhancement is $\mathrm{H}=3.5$.

\section{Discussion}

We have identified a self-consistent scenario for the simultaneous increase in beta and confinement in steady-state conditions. Because the theory of transport in tokamalis is not sufficiently developed to complete predictive simulations, we have adopted a strategy in which we maintain those features which we have observed experimentally to be important for high confinement. We have then modified the current density (or $q$ ) profile in a manner consistent with the features of high confinement and consistent with the transport and resultant bootstrap current, to increase the stability limit. The result is a configuration which we have called the second stable core VH-mode. This configuration combines the essential features of two separate experimental high performance operational modes first observed on DIII-D, the second stable core high beta discharges, $\mathrm{VH}$-mode discharges, and $\mathrm{q}_{0}>2$ of high $\beta_{\mathrm{p}}$ discharges.

There are a number of features that we have found to be required to maintain the highest confinement in DIII-D discharges. These are strong shaping , especially high triangularity; sheared rotation or sheared $E x B$, and finite edge pressure gradient and finite edge current density. The negative central shear region and higher $q(0)$ are clearly beneficial to increasing $\beta$ and we believe from experimental results is favorable for high confinement. All of these features are maintained in the second stable core VH-mode.

We believe the plasma rotation is very important for both confinement and stability, and means to maintain the rotation and rotational shear should be taken into serious consideration in tokamak designs. The beneficial impact of sheared $E x B$ flow on transport both theoretically and experimentally has been documented in a number of excellent papers. The results of independently controlling the rotation with external fields shows more clearly that the sheared $E x B$ plays a major role in reducing transport. It is clear from the results shown here that maintaining the mode rotation is essential for high beta operation in DIII-D; almost all discharge terminations in DII-D are a consequence of a non rotating low $\mathrm{n}$ mode. The emerging theory of the interaction of the plasma rotation and a resistive wall indicate that plasma rotation is essential (Bondeson 1994, Jensen 1983). The importance of rotation in achieving high performance, especially in achieving high beta, requires more emphasis and support for research in this area.

The improvement in the efficiency of tokamak reactors (measured by the cost of electricity) as a consequence of a simultaneous increase in the beta limit and the confinement has clearly been shown (Galambos 1994). We have identified a scenario for which both beta and confinement can be increased, consistent with steady-state conditions; i.e. fully penetrated current density profiles. The key to the increase in the beta limit is the negative central shear with $q_{\min }$ slightly larger than small rational numbers, $q_{\min }>1.0,1.5,2.0,2.5$, etc. And the key to maintaining the appropriate $q$ profile is current profile control with localized current drive. The simulations show ECCD is very attractive for controlling the current in both present day experiments (DII-D SSC VH-mode), and reactor plasmas. We believe that the attractiveness of these profiles for increasing both confinement and beta in reactor plasmas using several different transport models warrants serious consideration and should lead to an active research effort in current drive and current profile control. 
Future experiments in DIII-D are planned to explore active control of these advanced regimes to pulse lengths up to $10 \mathrm{~s}$ : experiments on TPX will extend them to steady state (Goldston, 1994).

\section{Acknowledgments}

This work represents the input of a large number of people, too numerous to mention: there contributions are gratefully acknowledged and much appreciated. The second stable core VH-mode scenario, specifically the inclusion of negative central shear at high $q_{\min }(>2)$ to increase stability, was developed in late 1991 and early 1992 during our DOE contract renewal effort. The encouragement and support from Drs. V.S. Chan, T.C. Simonen, and D. Overskei in pursuing advanced physics concepts to improve the tokamak reactor as a major DIII-D program element was essential in defining and developing the second stable core VH-mode scenario. A productive scientific interchange with TPX Physics Team is gratefully acknowledged, especially useful discussions with Dr. R. Goldston. This is a report of work supported by the U.S. Department of Energy under Contract Nos. DE-AC03-89ER51114 and DE-AC05-84OR21400.

\section{References}

Bernard L C, et al 1981 Comput Phys. Commun. 24377

Biglari H, et al 1990 Phys. Fluids B 21

Burrell K H, et al. 1992 Plasma Phys. and Contr. Fusion 341859

Burrell K H, et al. 1994 Phys. Plasmas 11536

Bondeson A, Ward D J 1994 Phys. Rev. Lett. 722709

Casper 1994 this conference

Chan V S, et al. 1993Proc. Int. Sherwood Fusion Theory Conf., Newport, paper IC26

Chance M S, et al.1987 Theory of Fusion Plasmas, Varenna (Editrice Compositori, Bologna, 1987) 87

Chance M S, et al. 1991 Plasma Phys. and Contr. Nucl. Fusion Research 1990 Washington (International Atomic Energy Agency, Vienna) vol 2 p 87

Chiu S C, et al. 1989 Nucl. Fusion 292175

Ferron J R, et al. 1993 Phys. Fluids B 52532

Ferron J R 1994 this conference.

Friedberg J P 1987 Ideal Magnetohydrodynamics (Plenum Press, New York, 1987)

Galambos ,et al. ORNL Ridge National Laboratory Report ORNLTN 12483 submitted to Nucl. Fusion

Gerver M, et al. 1988 Phys. Fluids 312674

Gohil P, et al. 1992 Fusion Eng (Proc. 14th IEEE/NPSS, California, 1991) vol 2 IEEE New Jersey 1199

Goldston R 1984 Plasma Phys. and Contr. Fusion 2687

Goldston R 1994a this conference

Greenfield C M, et al. 1993 Plasma Phys. and Contr. Fusion 35 B263

Greenwald M, et al. 1988 Nucl. Fusion 282199

Groebner R J 1993 Phys. Fluids B 52343

Harvey R W, et al. 1994 APS; Harvey R W, et al 1994 Proc. Int. Sherwood Fusion Theory Conf., Dallas, paper $2 \mathrm{C} 52$

Hender T C. et al. 1989 Nucl. Fusion 291279

Hoang G T, et al.1994 Nucl. Fusion 3475

Howl W, et al. 1992 Phys. Fluids B 41724

Hugon M, et al. 1992 Nucl. Fusion 3233

Hyatt A W 1994 this conference.

Ishida 1993 Plasma Phys. and Contr. Nucl. Fusion Research 1992 Würzburg (International Atomic Energy Agency, Vienna) vol $1 \mathrm{p} 219$

Jackson G L, et al. 1991 Phys. Rev. Lett. 673098

Jacquinot 1993 Plasma Phys. and Contr. Nucl. Fusion Research 1992 Würzburg (International Atomic Energy Agency, Vienna) vol $4 \mathrm{p} 1$

Jensen T H, Chu M S 1983 J Plasma Phys. 3057

JET Team 1993 Plasma Phys. and Contr. Nucl. Fusion Research 1992 Würzburgg (International Atomic Energy Agency, Vienna) vol 1 p 329

Kikuchi M, et al. 1990 Nucl. Fusion 30343

Kikuchi M, et al. 1993 Plasma Phys. Contr. Fusion 35 B39

Kessel C 1994 Phys. Rev. Lett. 721212

Lao L L, et al. 1993a Phys. Rev. Lett. 703435

Lao L L, et al. 1993b Plasma Phys. and Contr. Nucl. Fusion Research 1992 Würzburg (International Atomic Energy Agency, Vienna) vol 1 p 565 
Lao L L 1992 Phys Fluids B 4232

Lao L L, et al. 1989 Plasma Phys. and Contr. Nucl. Fusion Research 1988 Nice (International Atomic Energy Agency, Vienna) vol 1 p 83

La Haye R J, et al. 1994 submitted to Phys. Rev. Lett, General Atomics Report GA-A21544 1993

Lazarus E A, et al. 1991 Phys. Fluids B 32220

Lazarus E A, et al. 1992 Phys. Fluids B 43644

Lazarus E A, et al. 1993 Bull. Am. Phys. Soc. 381936

Lazarus E A, et al. to be published in 1994 Plasma Phys. and Contr. Nucl. Fusion Research 1994 Madrid (International Atomic Energy Agency, Vienna)

Matsuda K 1989 IEEE Trans. on Plasma Sci 176

Mauel 1993 Plasma Phys. and Contr. Nucl. Fusion Research 1992 Würzburg(International Atomic Energy Agency, Vienna) vol 1 p 205

Miller R L, et al. 1994 Proc. Int. Sherwood Theory Conf., Dallas invited paper 1B2

Osborne T H 1994 submitted to Nucl. Fusion; Osborne T H, et al to appear in Plasma Phys. and Contr. Fusion 1994

Politzer P A, et al.1994 Phys. Plasmas 11545

Ramos J J 1991 Phys. Lett. A 15265

Rebut P H, et al. 1989 Plasma Phys. and Contr. Nucl. Fusion Research 1988 Nice (International Atomic Energy Agency, Vienna) vol 2 p 191

Schissel D P 1991 Nucl. Fusion 3173

Shaing K C, et al. 1990 Phys. Fluids B 21492

Simonen T C, et al. 1988 Phys. Rev. Lett. 611720

Staebler G M, et al. 1994 Phys. Plasmas 1909

Strait E J, et al. 1993 Contr. Fusion and Plasma Phys., Lisbon (European Physical Society) vol 1 p 211

Strait E J 1994a Phys. Plasmas 11415

Strait E J 1994b this conference

Strait E J, et al. "Wall Stabilization of High Beta Tokamak DDischarges in DII-D," General Atomics Report GS-A21797, to be submitted to Phys. Rev. Lett.

St John H, et al. 1993 Contr. Fusion and Plasma Phys., Lisbon (European Physical Society) vol 1 p 99

Taylor T S, et al. 1991 Plasma Phys. and Contr. Nucl. Fusion Research 1990 Washington (International Atomic Energy Agency, Vienna) vol 1 p 177

Taylor T S, et al. 1993 Plasma Phys. and Contr. Nucl. Fusion Research 1992 Würzburg International Atomic Energy Agency, Vienna) vol 1 p 167

Troyon F, et al. 1984 Plasma Phys. and Contr. Fusion 26209

Tubbing B, et al. 1991 Nucl. Fusion 31839

Turnbull A D, et al. 1986 J. Comp. Phys. 66391

Turnbull A D, et al.1989 Nucl. Fusion 29629

Turnbull A D, et al 1994a Proc. Int. Sherwood Theory Conf., Dallas invited paper 2B1; to be pubnlished 1994 LAEA

Turnbull A D, et al. 1994b submitted to Phys. Rev. Lett.

Wesson J A 1978 Nucl. Fusion 1887

Wesson J A 1987 Tokamaks (Clarendon Press, Oxford) p 85

Wroblewski D, Lao L L 1993 Rev. Sci. Instrum. 635140

Yushmanov P N, et al. 1990 Nucl. Fusion 301999

Zarnstorf 1993 Plasma Phys. and Contr. Nucl. Fusion Research 1992 Würzburg (International Atomic Energy Agency, Vienna) vol 1 p 111 